\title{
Anhidrotic Ectodermal Dysplasia : A Case Report
}

\author{
Aprilin Krista Devia ${ }^{a}$, Cita Rosita Sigit Prakoeswa ${ }^{\mathrm{b}}$ \\ aprilindevi@gmail.com

\begin{abstract}
${ }^{\text {a }}$ Resident, Dermatology and Venereology Department Faculty of Medicine Universitas Airlangga, Dr. Soetomo Teaching Hospital Jl. Prof. dr. Moestopo 6-8, Surabaya 60286, Indonesia

${ }^{\mathrm{b}}$ Dermatologist, Dermatology and Venereology Department Faculty of Medicine Universitas Airlangga, Dr. Soetomo Teaching Hospital
\end{abstract} \\ J1. Prof. dr. Moestopo 6-8, Surabaya 60286, Indonesia
}

\begin{abstract}
Background: In the ectoderm-derived structures, anhidrotic ectodermal dysplasia (EDA) may lead to developmental abnormalities. An additional set of symptoms, including hypotrichosis, hypodontia, and anhidrosis, are also present. The diagnosis of EDA may be validated by genetic testing. The only way to control EDA is to provide parents with knowledge and counseling on how to keep their children safe from overheating. Early detection of EDA is critical so that the patient may get the most effective therapy.

Case: Herewith, we report A 6-year-old-girl with suspected EDA and was planned to perform Iodine-starch test. Her parent complained about dry skin, inability to sweat adequately since birth and episodes of fever. Physical examination showed dry skin, frontal bossing, sparse and fine of scalp hair and eyebrows, palmoplantar hyperkeratosis and peg shaped teeth. Genetic testing could not be done because limited of facility. Parents refused biopsy examination and Iodine-starch test revealed anhidrosis as high as the cervical myelum segment 5 and below.

Conclusion : Hypotrichosis, hypodontia, and anhidrosis are clinical triad of EDA. Genetic testing should be performed to establish a definitive diagnosis of EDA. If genetic testing facility not available, additional examination such as biopsy and Iodine test can be performed to support the diagnosis of EDA.
\end{abstract}

Keywords: Ectodermal dysplasia; biopsy; iodine test

\section{Introduction}

One set of hereditary illnesses called ectodermal dysplasias (EDs) includes a wide range of conditions that share developmental problems with at least two of the most well-known features that are thought to originate from the embryonic ectoderm: hair, teeth, nails, sweat glands, and others. Developmental disorders involving only one type of ectodermal structure, even if associated with other congenital malformations, are not classified as EDs. There are almost 200 conditions classified as Eds, and given the varied clinical presentations and clinical overlap, it can be difficult to diagnose them precisely (Nieman \& Grange, 2019).

When hypotrichosis is combined with one of the other ED symptoms (such as oligo or anodontia or hypo or anhidrosis), it is known as anhidrotic ectodermal dysplasia (EDA). EDA affects around one in every 5,000-10,000 babies (Anbouba, Carmany \& Natoli, 2020). It is most common in young children that a lack of sweat glands may result in life-threatening hyperthermia. Ectodysplasin A (EDA), a signaling protein, is lost or dysfunctional in people with EDA, a condition caused by X-chromosomal EDA gene variations. Gene variations that have been disclosed so far include more than 200 distinct null mutations (Wohlfart et al, 2020). 
The diagnosis of EDA is recognized readily when expected, such as when a child is born into family with a known history of EDA. However, without advance knowledge, the diagnosis can initially be clinically challenging. As the patient ages, the characteristic features of hypotrichosis, hypohidrosis, and hypodontia become more evident. Hypoplastic alveolar ridges, indicating lack of teeth, can be early diagnostic clue. Although rarely necessary, evaluation of sweating by examination for sweat pores with iodine solution, quantification of pilocarpine-induced sweating, or skin biopsy to assess for the absence of eccrine structures in the scalp and or palmar region may help confirm a diagnosis (Nieman \& Grange, 2019). We reported the case of anhidrotic epidermal dysplasia. This case aims to help confirm a diagnosis of EDA, and also performing iodine solution as an important additional examination to establish diagnosis of EDA if biopsy can not perform.

\section{Case presentation}

A 6-year-old girl presented to the Dermatovenereology Outpatient Clinic at Soetomo General Hospital with the complaint of dry skin and inability to sweat adequately since at birth. The parent revealed that the child had intermitten episodes of fever in the past. Such episodes used to occurred more frequently in hot climate, but no definite cause had been diagnosed. She had episodes of seizure since 1,5 year old. Parent also complaint delayed eruption of abnormally shaped teeth. The child had problem with hearing and delay speech development. Parent denied a consanguineous marriage or having a family member with similar features as the child. No history of birth complication during delivery.

When she was weighed, her pulse rate was 70 beats per minute, her respiration rate was 20 beats per minute, and her body temperature was 36.9 degrees Celsius, all of which were within the normal range for her age and health condition. Dyspnea, cyanosis, or anemia were not detected during the patient's head and neck assessment. The lungs, heart, and thorax were all found to be in good health. This person had no feeling in any of his organs, including his stomach, liver, or spleen. On palpation, the arms and legs exhibited no signs of edema and were warm.

The results of the dermatologist's examination revealed that the patient had dry skin. In the presence of frontal bossing, Hair on the scalp was thin, fine, golden, and without eyebrows. The nails were found to be normal. There was hyperkeratosis of the palms and soles. A hypodontia diagnosis and peg-shaped teeth were made during the examination. 


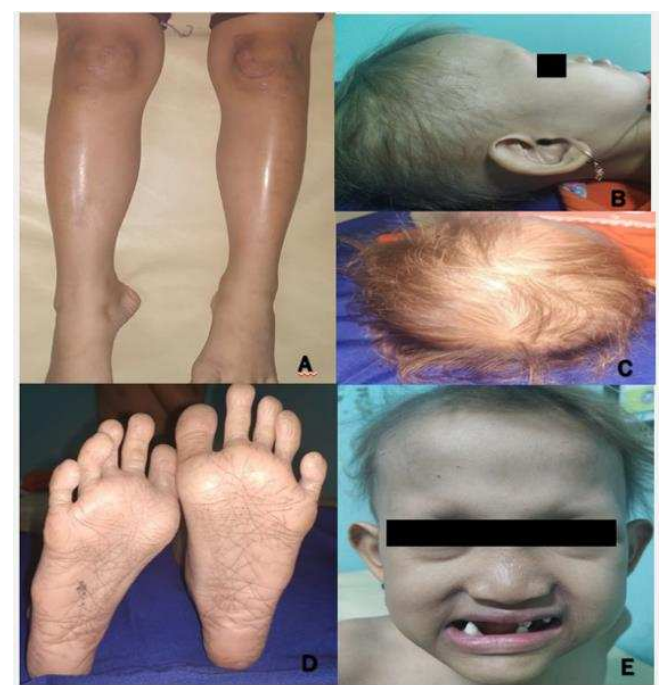

Fig 1. Clinical photograph of the legs showing dry skin (a), frontal bossing (b), sparse, fine, and blonde hair (c), palmoplantar hyperkeratosis (d), hypodontia and peg shaped teeth (e).

FT4 and TSH examination had normal result. The child was advised to had a biopsy, but the parent refused. The patient was consulted to for pediatric and neurology department for further evaluation. Iodine test by neurology department showed anhidrosis as high as the cervical myelum segment 5 and below. Genetic testing could not be done.

The patient was treated with atopiclair as emmolient. The youngster was diagnosed with EDA based on the child's history, clinical characteristics, and physical examination.

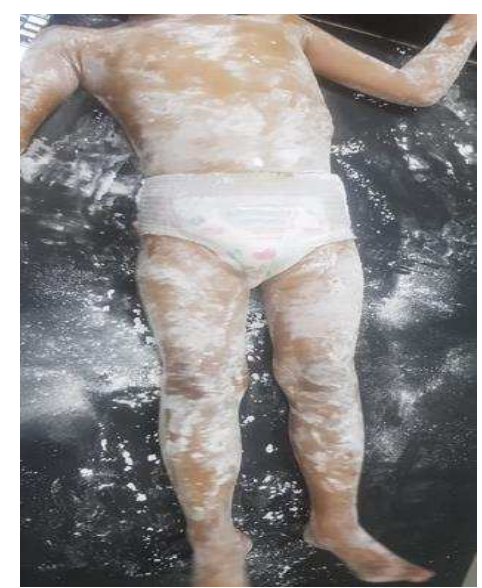

Fig 2. Iodine test showed anhidrosis as high as the cervical myelum segment 5 and below. 


\section{Discussion}

It is estimated that around one in every 100,000 newborns are affected by EDs, which are a set of genetic illnesses that are caused by primary developmental abnormalities of two or more embryonic ectoderm components (Bhakta, Barthunia, Nigam \& Pawar, 2019). There are three symptoms that define EDA: hair loss (hypotrichosis), missing or partially missing teeth (anodontia or hypodontia), and reduced sweating (anhidrosis or hypohidrosis) (Chandravanshi, 2019). Hypotrichosis, hypodontia, and anhidrosis were all present in this infant, indicating EDA.

Ectodysplasin signal transduction pathway mutations are the cause of EDA. During embryogenesis, this cascade is activated inside epithelial cells. Eccrine sweat glands, teeth, and hair follicles are all involved in the morphogenesis of the target genes. The ectodysplasin ligand, encoded by the EDA gene, starts signaling (Sen, Das, Sharma, 2020). Because to a lack of resources, genetic testing could not be performed on the kid.

Classical characteristics are frequently evident by the time a child is only a few months old. Most have a pathognomonic facial appearance that includes a square forehead, broad nostrils, wide cheekbones with flat malar ridges, a thick everted lower lip, and a prominent chin. Skin is fine, smooth, and dry in both children and adults (Asai, 2011). The disease is characterized by darkening of the periorbital region and fine lines and wrinkles around the eyes (Paller, 2016). Patients with periorbital wrinkling, dry skin, and frontal bossing were seen. Six percent of infants with high fevers and feeding problems have a febrile seizure as a result of their illness (Srinithi, Ramya, Sundari, 2021). Anhidrosis is the most prevalent cause of unexplained pyrexia and heat sensitivity (Bhakta, Barthunia, Nigam \& Pawar, 2019). This is in line with what the youngster has really encountered. This patient has normal nails. Most people's nails are in good health. Some individuals have been reported to have thin, brittle nails with longitudinal ridges (Asai, 2011). Teeth with peg-shaped or conical incisors are more common, however dental abnormalities may range from full absence to partial absence. With peg-shaped teeth, the patient developed hypodontia.

EDA may be diagnosed clinically in individuals with the complete syndrome, however partial instances and carriers of the illness might be difficult to diagnose. In these circumstances, more testing is required to show a drop-in sweating or a decrease in the number of eccrine glands. There are a few tests that may be used to confirm whether or not someone has sweat gland dysfunction, including the minor test and the iodide-starch test. Exams may help distinguish between females with X-linked EDA carriers and those with autosomally transmitted EDA, a condition in which sweat gland activity is almost non-existent (Martin, 2013). As far as the cervical myelum segment 5 and below, this patient's iodine-starch test result indicated anhidrosis from the test. EDA is frequently diagnosed without a skin biopsy. A lack of or partial development of the eccrine glands may 
be discovered with this procedure. The number of sebaceous glands and hair follicles is also reduced (Liu, 2012). This patient's parents were unwilling to allow a skin biopsy to be done.

For ED, there is currently no effective medication available. Patients are treated in accordance with the ectodermal structures implicated in their condition (Hasan, 2019). Educating parents and providing them with advice on how to prevent their children from being overheated are two important components of EDA management. Hyperthermia may be avoided by keeping the patient in a cold, dark place. Wearing light clothes and refraining from strenuous physical activity are recommended (Liu, 2012). The patient was instructed to drink plenty of fluids and wear light clothes to stay hydrated and regulate her body temperature. Basic measures such as use of skin emollients are employed. We gave atopiclair as emollient to patient for the dry skin. Regular dental evaluation should begin early, so we referred patient to dentistry department. Patient also reffered to pediatric department for further evaluation.

A dermatologist, a pediatrician, and a dentist can all provide excellent therapy for an ED patient if they recognize the condition early enough (Chandravanshi, 2019).

\section{Conclusion}

This patient was diagnosed with EDA based on history of inability of sweat adequately, intermitten episodes of fever, delayed eruption of abnormally shaped teeth, clinical features of hypotrichosis, hypohidrosis and hypodontia. Physical examination we found dry skin, frontal bossing, sparse and fine of scalp hair and eyebrows, palmoplantar hyperkeratosis and also peg shaped teeth. Iodide-starch test revealed anhidrosis as high as the cervical myelum segment 5 and below.

It's important that dermatologists, pediatricians, and dentists be aware of this condition since they're the first people to check patients. Genetic testing could be done to confirmed the diagnosis of EDA, but in limited facility, we recommeded additional examination such as iodine test if biopsy also could not be done.

\section{Acknowledgment}

Thanks to all of the dermatology and venereology lecturers at Airlangga University's faculty of medicine, my family and friends, for their constant encouragement and prayers.

\section{References}

Anbouba GM, Carmany EP, Natoli JL. 2020. The characterization of hypodontia, hypohidrosis, and hypotrichosis associated with X-linked hypohidrotic ectodermal dysplasia: A systematic review. American Journal of Medical Genetics Part A, 20(2): 1-11. 
Asai Y, Irvine AD. Ectodermal dysplasias. 2011. In: Irvine A, Hoeger P, Yan A, editors. Harper's Textbook of Pediatric Dermatology. $3^{\text {rd }}$ ed. UK: Blackwell Publishing Ltd. p. 127.69.

Bhakta P, Barthunia B, Nigam H, Pawar P. 2019. Ectodermal dysplasia-A rare case report. J Family Med Prim Care, 8(9): 3054-3056.

Chandravanshi, SL. 2019. Hypohidrotic ectodermal dysplasia: a case report. Orbit, 39(1): 1-4.

Hasan S, Govind M, Sawai MA, Ansari MD. 2019. Hypohidrotic ectodermal dysplasia with autosomal recessive inheritance pattern: Report of a rare and unusual case with a brief review of literature. J Oral Maxillofac Pathol, 23(3): 479 .

Liu KC, Huang CY, Chao SC. 2012. Anhidrotic ectodermal dysplasia-A case series in a medical center in southern Taiwan. Dermatologica Sinica, 30(2): 39-42.

Martin PG, Martin AH, Torrelo A. 2013. Ectodermal dysplasia: A clinical and molecular review. Actas DermoSifiliográficas, 104(6): 451-470.

Nieman EL, Grange DK. 2019. In: Kang et al, editors. Fitzpatrick’s Dermatology. $9^{\text {th }}$ Edition.

Paller AS, Mancini AJ. 2016. Hurwitz Clinical Pediatric Dermatology. Fifth ed. Canada: Elvesier. p. 147.

Sen S, Das S, Sharma S. 2020. Complete phenotypic expression of hypohidrotic ectodermal dysplasia in a female patient. Indian J Dermatol, 65(1): 540-1.

Srinithi R, Ramya R, Sundari S. 2021. Hypohidrotic ectodermal dysplasia: a rare inherited multisystem disorder. Indian Journal of Experimental Biology, 59(10): 751-753.

Wohlfart S, Meiller R, Hammersen J, Park J, Menzel-Severing J, Melichzr VO, et al. 2020. Natural history of X-linked hypohidrotic ectodermal dysplasia: a 5-year follow-up study. Orphanet J Rare Dis, 15(1): 7. 